\title{
Sweetened beverage intake and risk of latent autoimmune diabetes in adults (LADA) and type 2 diabetes
}

\author{
Josefin E Löfvenborg', Tomas Andersson 1,2, Per-Ola Carlsson ${ }^{3}$, Mozhgan Dorkhan $^{4}$, \\ Leif Groop ${ }^{4}$, Mats Martinell' ${ }^{5}$, Tiinamaija Tuomi ${ }^{6}$, Alicja Wolk ${ }^{1}$ and Sofia Carlsson' \\ ${ }^{1}$ Institute of Environmental Medicine, Karolinska Institutet, Stockholm, Sweden, ${ }^{2}$ Center for Occupational and \\ Environmental Medicine, Stockholm County Council, Stockholm, Sweden, ${ }^{3}$ Department of Medical Sciences, \\ Uppsala University, Uppsala, Sweden, ${ }^{4}$ Department of Clinical Sciences, Lund University, Malmö, Sweden, \\ ${ }^{5}$ Department of Public Health and Caring Sciences, Uppsala University, Uppsala, Sweden, and ${ }^{6}$ Endocrinology, \\ Abdominal Centre, Helsinki University Hospital, Research Program for Diabetes and Obesity, University of Helsinki, \\ and Finnish Institute for Molecular Medicine, University of Helsinki, Helsinki, Finland
}

Correspondence should be addressed to J E Löfvenborg Email

josefin.lofvenborg@ki.se

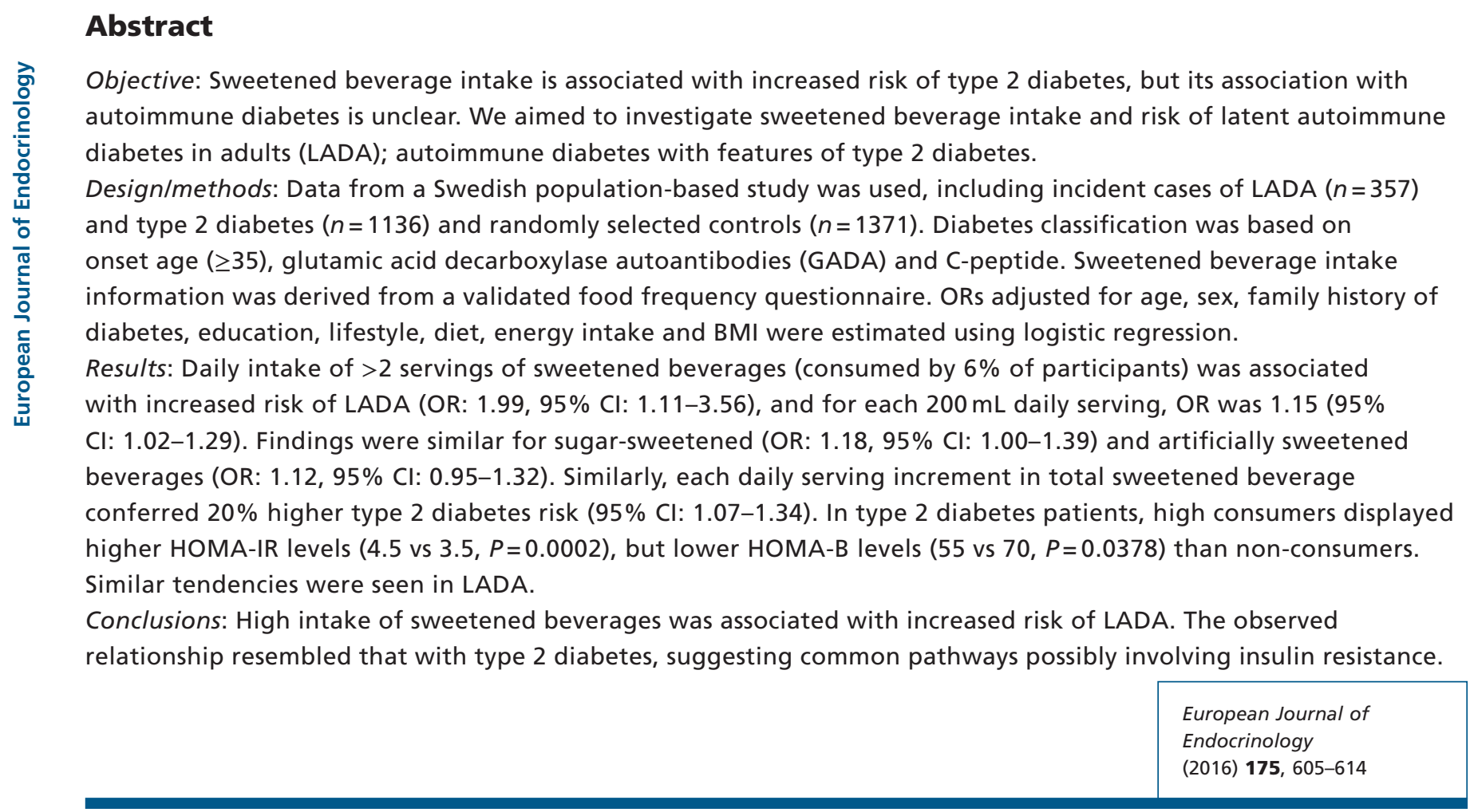

\section{Introduction}

Sugar-sweetened beverage intake has been linked to increased risk of type 2 diabetes in numerous studies $(1,2,3,4,5,6)$; the most recent meta-analysis found that the risk increases by $13 \%$ for an increment of each serving per day in intake (7). Suggested routes by which sugar-sweetened beverages may influence diabetes risk are through glycemic or insulinemic responses or by
() 2016 European Society of Endocrinology Printed in Great Britain contributing to excessive energy intake $(8,9)$. Artificially sweetened beverages have also been associated with an increased risk of type 2 diabetes $(1,10)$, although they contain no calories and are thus adding neither to the glycemic load nor to the total daily energy intake (11). Suggested mechanisms related to artificially sweetened beverages include enhanced appetite by distortion of

Published by Bioscientifica Ltd. 
satiety signaling (11) and deteriorated glucose tolerance provoked by alterations in gut microbiota (12).

The association between sweetened beverages and autoimmune diabetes is less clear $(13,14)$, but a recent study suggests that intake of sugar-sweetened beverage may increase the risk of type 1 diabetes in genetically susceptible individuals (13). Possible mechanistic pathways for its involvement in autoimmune processes include induced beta cell apoptosis (15), which could be due to toxic levels of glucose (16), induced oxidative stress (17) or an overloaded beta cell possibly leading to it being more visible to the immune system (18).

Latent autoimmune diabetes in adults (LADA) is a hybrid form of diabetes with features of both type 1 and type 2 diabetes. In addition to sharing autoimmune markers (typically glutamic acid decarboxylase antibodies (GADA)) with type 1 diabetes patients, LADA patients also present with (mild to moderate) insulin resistance, a feature shared with type 2 diabetes (19). Thus, it is possible that intake of sweetened beverages may influence the risk of LADA through mechanisms related to either autoimmune processes or insulin resistance, but this remains to be explored. LADA accounts for $9 \%$ of all adultonset diabetes (20) and therefore constitutes an important part of the diabetes spectrum. Still, potential risk factors remain largely unexplored.

We aimed to investigate the risk of LADA and type 2 diabetes in relation to intake of sugar-sweetened and artificially sweetened beverage and to explore how it relates to markers of insulin resistance, beta cell function and autoimmunity.

\section{Subjects and methods}

\section{Study population and design}

Analyses were based on data from the ESTRID study (Epidemiological Study of Risk Factors for LADA and Type 2 Diabetes), a Swedish population-based case-control study initiated in 2010 (Supplementary Fig. 1, see section on supplementary data given at the end of this article) (21). ESTRID is a sub-study to ANDIS (All New Diabetics in Scania; http://andis.ludc.med.lu.se), which is a large study aiming to characterize all new diabetes cases within the county of Scania (southern Sweden, 1.3 million inhabitants) with regard to diabetes type, clinical features and genotype. LADA constitutes 5\% of all diabetes in the ANDIS Registry, all ages included (22). All newly diagnosed cases of LADA identified in ANDIS since 2010 have been invited to participate in the ESTRID study, together with a random sample of incident type 2 diabetes cases in ANDIS (4:1 ratio to LADA cases). Since 2012, ESTRID has also recruited cases of LADA and type 2 diabetes from ANDiU (All New Diabetics in Uppsala; http://www.andiu.se/), a study using the ANDIS setup but covering the county of Uppsala (in mid-Sweden, 350000 inhabitants). The Swedish Population Register was used to select a random sample of controls who was free of diabetes, aged $\geq 35$ years and matched to the cases based on participation date and county (incidence density sampling (23)) in a 6:1 ratio to LADA cases. A self-administered questionnaire including a large number of questions on health and lifestyle factors was sent by mail to all cases and controls. Participants included in this study were recruited in ESTRID between September 2010 and July 2015. The participation rate was $81 \%$ for cases and $66 \%$ for controls.

The current analysis excluded those with incomplete information on exposure $(n=26)$ or any of the main covariates (family history of diabetes, education, physical activity, smoking, alcohol intake and BMI; $n=53)$. We further excluded individuals with reported total daily energy intake that deviated more than 3 s.D. from the $\log _{\mathrm{e}}$-transformed sex-specific mean energy intake $(n=7$ women and $n=11$ men). After exclusions, 357 cases of LADA, 1136 cases of type 2 diabetes and 1371 controls remained for analysis $(96 \%$ of included cases were recruited from ANDIS and 4\% from ANDiU). Informed consent was provided by all participants. The study has been approved by the Regional Ethical Review Board at Karolinska Institutet, Stockholm, Sweden.

\section{Serological assays}

C-peptide was measured by the Cobas e 601 analyzer (Roche Diagnostics) or IMMULITE 2000 (Siemens Healthcare Diagnostics Product). GADA was quantitatively determined using ELISA (enzyme immunoassay; RSR Limited, Cardiff, UK). ELISA gives a maximum value of $250 \mathrm{IU} / \mathrm{mL}$ and has a sensitivity of $84 \%$ and specificity of $98 \%$ for a cutoff level of $10.7 \mathrm{IU} / \mathrm{mL}$ (24).

\section{Case definition}

All diabetes cases were diagnosed by a physician within the regional health care system. Classification into diabetes subtype was based on fasting blood samples collected at the time of diagnosis. Criteria for LADA diagnosis were age $\geq 35$ years, GADA positivity ( $\geq 10 \mathrm{IU} /$ $\mathrm{mL}$ ) and serum C-peptide exceeding the lower limit 
for the normal range as specified by the manufacturer $(\geq 0.2 \mathrm{nmol} / \mathrm{L}$ for IMMULITE and $\geq 0.3 \mathrm{nmol} / \mathrm{L}$ for Cobas). Type 2 diabetes criteria were $\geq 35$ years, GADA negativity $(<10 \mathrm{IU} / \mathrm{mL})$ and C-peptide levels above $>0.6 \mathrm{nmol} / \mathrm{L}$ (IMMULITE) or $>0.72 \mathrm{nmol} / \mathrm{L}$ (Cobas). There are no unified criteria for LADA diagnosis and classification, but the criteria used in this study are in line with previous literature (19). The exception is C-peptide, which in this study is used as an indicator of remaining insulin production to separate LADA from type 1 diabetes. It replaces the insulin therapy criterion (i.e. no insulin therapy within 6-12 months of diagnosis) that is commonly used, but to some extent, open to subjectivity because it reflects the assessment made by the treating physician. Homeostatic Model Assessment for insulin resistance (HOMA-IR) and beta cell function (HOMA-B) were calculated based on fasting plasma glucose and C-peptide levels at diagnosis (25).

\section{Dietary assessment}

Information on sweetened beverage intake was obtained from a semi-quantitative Food Frequency Questionnaire (FFQ) consisting of 132 items. The participants were asked to report their habitual food intake during the preceding year. The cases were specifically instructed to report their average intake during the year before diagnosis. Four questions were asked about sweetened beverage intake: cola, other soft drinks/soda, diet cola and other diet soft drinks/soda. The participants were asked to report their average number of $200 \mathrm{~mL}$ servings per day or per week. 'Other soft drink/soda' also refers to other sweetened beverages such as nectars and diluted syrups. The FFQ includes a separate question on $100 \%$ fruit juice, which was not evaluated in this study.

Information on potentially confounding dietary factors was also derived from the FFQ. Nutritional data from the Swedish National Food Agency database were used to estimate the average daily intakes of total energy and nutrients based on the FFQ.

The FFQ has been validated against 24-h recall interviews (26), diet records (27) and dietary biomarkers (28). Validation of individual food items against four 1 -week diet records in a subsample of 129 women indicated a correlation of 0.6 for FFQ-reported sweetened beverage intake (A Wolk, unpublished results). The FFQ-based nutrient estimates have been validated in comparison with the mean of fourteen 24-h recall interviews in 248 men, and showed Spearman correlation coefficients ranging from 0.44 (protein) to 0.81 (alcohol) (26).

\section{Other covariate assessment}

BMI at age 20 and at present was calculated based on self-reported height and weight. Education was assessed with a question of the highest attained level of education and categorized into elementary school, high school or university. Family history of diabetes was categorized as yes/no for any type of diabetes in first-degree relatives. Leisure time physical activity during the preceding year was assessed by a question with four response options ranging from sedentary to regularly active. Smoking habits were categorized as never smoker, former or current smoker. Estimated total alcohol intake was categorized into non-consumption, $0.01-4.9 \mathrm{~g} /$ day, $5.0-14.9 \mathrm{~g} /$ day and $\geq 15.0 \mathrm{~g} /$ day.

\section{Statistical analysis}

Conditional logistic regression model was used to estimate the odds ratios (OR) and 95\% confidence intervals (CI) of LADA and type 2 diabetes in relation to sweetened beverage intake. We analyzed not only the total intake but also performed separate analysis for sugar-sweetened and artificially sweetened beverages. One serving was defined as $200 \mathrm{~mL}$, and exposure was assessed as continuous as wells as categorical $(<1,1-2$ and $>2$ servings per day) with non-consumers as reference. ORs are presented after adjustment for age and sex (model 1), and with additional adjustment for education, family history, physical activity, smoking, alcohol intake, total energy intake (kcal/ day) and the following dietary factors in grams per day: vegetables, fruit, whole grain, fatty fish, red/processed meat, biscuits/sweets/salty snacks and coffee (servings/ day) (model 2). Current BMI was considered to potentially be in the causal pathway and was included separately in the model (model 3). Results of model 3 are presented in the text, unless otherwise specified. Stratified analyses were performed using BMI and GADA levels (LADA) respectively. Sensitivity analyses included restricting the analysis to cases who participated within six months of diagnosis and to those not being on dietary treatment to minimize reverse causation. To investigate whether the association between highly sweetened beverage consumption and diabetes reflects increased thirst before diagnosis, we assessed the association between water consumption and risk of LADA/type 2 diabetes.

Because of the incidence density sampling design, the estimated ORs could be interpreted as incidence rate ratios (23). Two-sided $P$ values were calculated using $\chi^{2}$ (proportions) or Wilcoxon-Mann-Whitney test 
(means and medians). All analyses were performed using SAS 9.4 (SAS Institute Inc., Cary, NC, USA).

\section{Results}

\section{Clinical characteristics}

Mean age was 58.8 years for LADA patients, 63.2 years for type 2 diabetes patients and 58.5 years for controls (Table 1). Patients with LADA had lower BMI and were less insulin resistant, but had worse beta cell function and were more often treated with insulin after diagnoses compared with type 2 diabetes patients. Family history of diabetes was almost as common in LADA (45\%) as in type 2 diabetes (50\%), but less frequent among controls (24\%). Median diabetes duration on the date of participation was 7.5 months for LADA and 5.3 months for type 2 diabetes (data not shown in table).

\section{Lifestyle characteristics by category of sweetened beverage consumption}

The proportion of participants (irrespective of case/ control status) who were non-consumers of both sugarsweetened and artificially sweetened beverages was $63 \%$. High consumers of total sweetened beverages ( $>2$ servings per day of sugar-sweetened beverages and artificially sweetened beverages) had considerably higher BMI than other consumers and the largest annual weight gain $(1.05 \%$ vs $0.72 \%$ in non-consumers) since age 20 (Table 2). In addition, they had generally less favorable lifestyle compared with those consuming little or no sweetened beverages, including higher prevalence of smoking, low physical activity, lower consumption of vegetables, fruit, fatty fish and whole grains and higher consumption of red/processed meat and biscuits/sweets/ salty snacks. Comparing high consumers of sugarsweetened vs artificially sweetened beverages suggests that the latter group reported lower total energy intake but tended to have higher BMI both at present and at age 20 (Supplementary Table 1).

\section{LADA and sweetened beverage consumption}

The risk of LADA was increased 2-fold (OR: 1.99, 95\% CI: 1.11-3.56) for the group consuming more than two servings of sweetened beverages per day compared with non-consumers (Table 3), whereas no excess risk was seen in consumers of up to two servings per day. At consumption levels exceeding five servings per day (7 cases/ 6 controls), an OR of 4.47 (95\% CI: 1.21-16.47) was observed. Each increment of daily serving conferred a 15\% increased risk (OR: 1.15, 95\% CI: 1.02-1.29). The results were similar in a more refined analysis of sugarsweetened and artificially sweetened beverage intake separately, with an OR of 1.18 (95\% CI: 1.00-1.39) and OR of 1.12, (95\% CI: 0.95-1.32) respectively, per daily serving.

Stratifying the analyses by sex, age, family history of diabetes, BMI and median GADA levels $(191 \mathrm{IU} / \mathrm{mL})$ of the LADA patients indicated that the excess risk in high consumers of sweetened beverages was consistent across most subgroups (Supplementary Fig. 2). However, the increased risk of LADA was primarily seen in men, but analysis of women was based on a very limited number of high consumers (6 cases/19 controls).

Table 1 Characteristics of LADA and type 2 diabetes cases from ANDIS/ANDiU and controls participating in ESTRID, $2010-2015$. Data are presented as mean (s.D.) unless otherwise stated.

\begin{tabular}{l}
\hline$n$ \\
\hline Age (years) \\
Sex (\% women) \\
BMI (kg/m²) \\
Overweight (BMI $\geq 25)(\%)$ \\
Obese (BMI $\geq 30)(\%)$ \\
Family history of diabetes (\% yes) \\
Insulin treatment (\%) \\
Metformin and/or sulfonylurea treatment $(\%)^{b}$ \\
HOMA-IR, median (interquartile range) \\
HOMA-B, median (interquartile range) \\
GADA, median (interquartile range) $(I U / m L)$
\end{tabular}

\begin{tabular}{c}
\hline Controls \\
\hline 1371 \\
$58.5(13.3)$ \\
53 \\
$25.9(4.0)$ \\
54 \\
14 \\
24 \\
- \\
- \\
- \\
- \\
-
\end{tabular}

\begin{tabular}{c}
\hline LADA \\
\hline 357 \\
$58.8(12.5)$ \\
47 \\
$28.2(5.4)$ \\
71 \\
32 \\
45 \\
42 \\
55 \\
$2.7(1.8-4.4)$ \\
$39(14-69)$ \\
$191(27-250)$ \\
\hline
\end{tabular}

\begin{tabular}{c}
\hline Type 2 diabetes \\
\hline 1136 \\
$63.2(10.3)$ \\
40 \\
$31.1(5.4)$ \\
93 \\
51 \\
50 \\
6 \\
62 \\
$3.5(2.7-4.9)$ \\
$68(42-92)$ \\
-
\end{tabular}

\begin{tabular}{r}
\multicolumn{1}{c}{$\boldsymbol{P}^{\mathrm{a}}$} \\
\hline \\
$<0.0001$ \\
0.0117 \\
$<0.0001$ \\
$<0.0001$ \\
$<0.0001$ \\
0.0813 \\
$<0.0001$ \\
0.0198 \\
$<0.0001$ \\
$<0.0001$ \\
-
\end{tabular}

${ }^{a} P$ for difference between LADA and type 2 diabetes. ${ }^{\text {b}}$ The information is only available for LADA and type 2 diabetes patients. 


\section{Type 2 diabetes and sweetened beverage consumption}

The OR of type 2 diabetes was increased by 2.39 (95\% CI: 1.39-4.09) in those consuming more than two servings of sweetened beverages per day compared with nonconsumers (Table 3). Consumption of more than five servings per day (18 cases/6 controls) was associated with an OR of 10.53 (95\% CI: 2.75-40.33). The risk of type 2 diabetes was increased by $20 \%$ for each daily serving increment (OR: 1.20, 95\% CI: 1.07-1.34). Similar findings were seen when assessing sugar-sweetened (OR: 1.21, 95\% CI: 1.05-1.41) and artificially sweetened beverages (OR: 1.18, 95\% CI: 1.01-1.38) separately. The excess risk in high consumers was seen across subgroups of sex, age, family history of diabetes and BMI (Supplementary Fig. 3).

\section{HOMA-IR, HOMA-B and GADA}

Median HOMA-IR tended to be higher among high consumers of sweetened beverages ( $>2$ servings per day) compared with that among non-consumers for LADA (3.7 vs $2.5, P=0.0561$ ), and more clearly shown for type 2 diabetes patients ( 4.5 vs $3.5, P=0.0002$ ). In contrast, there was a tendency of lower HOMA-B levels among high consumers compared with that among non-consumers in the LADA patient group ( 20 vs $48, P=0.0588$ for median). This difference was more pronounced among type 2 diabetes patients (55 vs 70, $P=0.0378$ ). No difference in GADA levels was observed in high consumers compared with that in non-consumers (250 vs $204, P=0.6511)$ among LADA patients.

\section{Sensitivity analyses}

The results were unchanged when using occupation instead of education as an indicator for socioeconomic status. Sensitivity analysis conducted to explore reverse causation indicated similar results; when restricting the analysis to patients responding to the questionnaire within six months of diagnosis, the excess risk among high ( $>2$ servings per day) consumers persisted for both LADA (OR: 2.74, 95\% CI: 1.16-6.47) and type 2 diabetes (OR: 2.02, 95\% CI: 1.09-3.74). For type 2 diabetes in which we had larger number of cases, we could further restrict the analysis to cases responding within three months and observed a similar association (OR: 3.18, 95\% CI: 1.13-8.95). Exclusion of patients reporting that they were on dietary treatment while filling out the questionnaire indicated similar results for LADA (OR: 2.01, 95\% CI: 1.07-3.78) and type 2 diabetes (OR: 2.15, 95\% CI: 1.21-3.83).

Table 2 Characteristics of participants in ESTRID by intake of sweetened beverages. Data are presented as mean (s.D.) unless otherwise stated.

\begin{tabular}{|c|c|c|c|c|c|}
\hline & \multicolumn{4}{|c|}{ Total sweetened beverage intake (no. of $200 \mathrm{~mL}$ servings/day) } & \multirow[b]{2}{*}{$P$} \\
\hline & 0 & $<1$ & $1-2$ & $>2$ & \\
\hline Mean intake (g/day) & 0 & 76 & 287 & 890 & (Highest vs lowest) \\
\hline$n(\%)$ & $1815(63)$ & $587(20)$ & $302(11)$ & $160(6)$ & \\
\hline Sugar-sweetened beverage (g/day) & $0(0)$ & $46(44)$ & $148(146)$ & $461(676)$ & $<0.0001$ \\
\hline Artificially sweetened beverage (g/day) & $0(0)$ & $30(44)$ & $139(153)$ & $429(424)$ & $<0.0001$ \\
\hline Age (years) & $63.0(11.4)$ & $55.2(11.8)$ & $58.6(13.6)$ & $52.6(11.6)$ & $<0.0001$ \\
\hline Sex (\% women) & 50 & 46 & 38 & 33 & $<0.0001$ \\
\hline High level of education (\% university) & 29 & 31 & 22 & 14 & $<0.0001$ \\
\hline BMI current $\left(\mathrm{kg} / \mathrm{m}^{2}\right)$ & $27.8(5.1)$ & $28.4(5.7)$ & $29.1(5.1)$ & $30.8(6.9)$ & $<0.0001$ \\
\hline BMI at age $20\left(\mathrm{~kg} / \mathrm{m}^{2}\right)^{\mathrm{a}}$ & $21.7(3.1)$ & $22.0(3.5)$ & $22.4(3.2)$ & $23.3(4.0)$ & $<0.0001$ \\
\hline Average yearly weight gain (\%) since age $20^{a}$ & $0.72(0.62)$ & $0.88(0.77)$ & $0.89(0.74)$ & $1.05(0.82)$ & $<0.0001$ \\
\hline Physically inactive $(\%)$ & 16 & 17 & 26 & 30 & $<0.0001$ \\
\hline Family history of diabetes (\% yes) & 37 & 39 & 31 & 42 & 0.1793 \\
\hline Smoking status (\% current) & 18 & 22 & 26 & 34 & $<0.0001$ \\
\hline Alcohol (g/day) & $8.8(9.8)$ & $8.8(9.4)$ & $7.5(10.2)$ & $6.9(9.1)$ & 0.0049 \\
\hline Coffee (servings/day) & $3.2(2.3)$ & $3.2(2.2)$ & $2.9(2.3)$ & $2.7(2.4)$ & 0.0043 \\
\hline Vegetables (g/day) & $218(146)$ & $205(115)$ & $196(159)$ & $157(110)$ & $<0.0001$ \\
\hline Fruits (g/day) & $162(128)$ & $146(112)$ & $153(124)$ & $115(116)$ & $<0.0001$ \\
\hline Red or processed meat (g/day) & $87(51)$ & $102(52)$ & $107(65)$ & $118(63)$ & $<0.0001$ \\
\hline Fatty fish (g/day) & $22(20)$ & $20(19)$ & $21(17)$ & $16(14)$ & $<0.0001$ \\
\hline Whole grain (g/day) & $56(34)$ & $51(30)$ & $52(32)$ & $45(33)$ & $<0.0001$ \\
\hline Biscuits, sweets, and salty snacks (g/day) & $31(26)$ & $42(30)$ & $45(32)$ & $50(38)$ & $<0.0001$ \\
\hline Energy (kcal/day) & $1762(557)$ & $1900(627)$ & $2129(766)$ & $2236(785)$ & $<0.0001$ \\
\hline
\end{tabular}




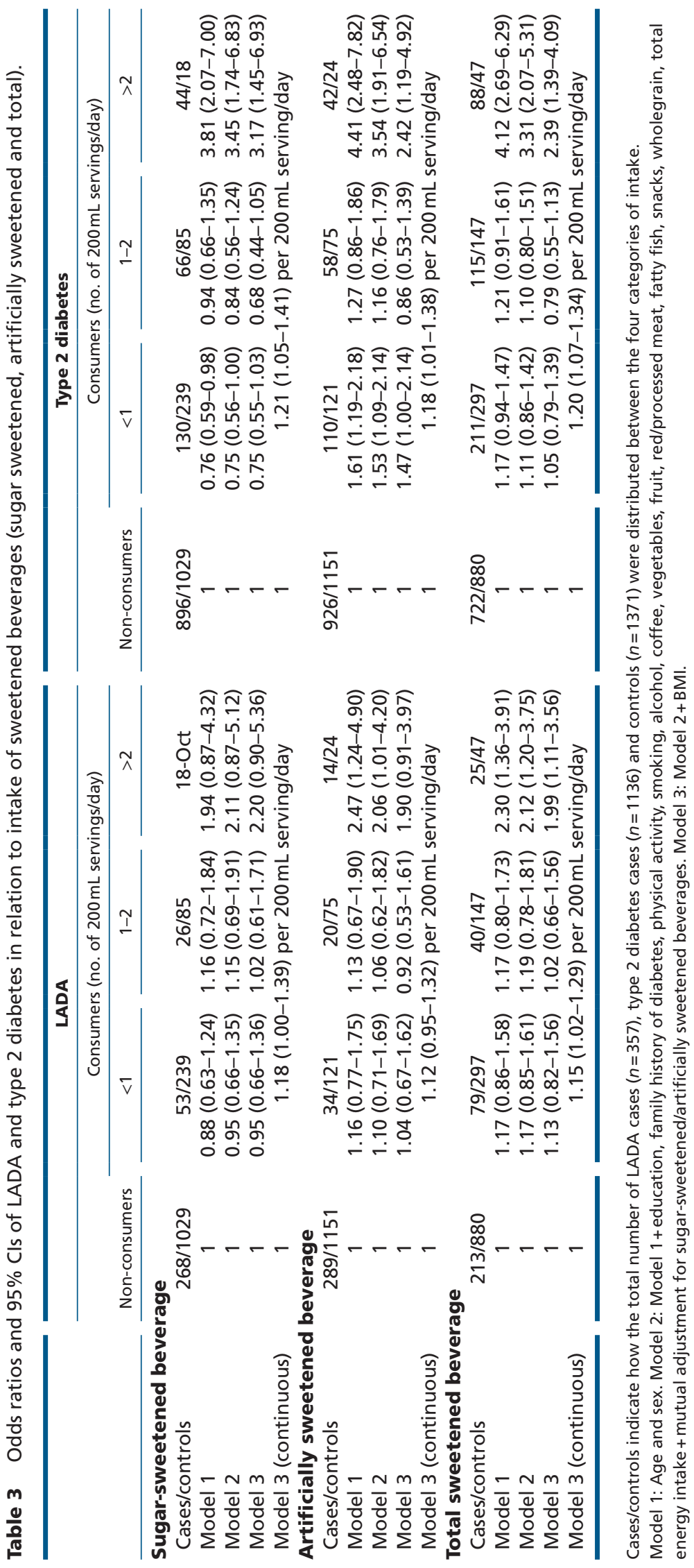


Finally, there was no association between water consumption (as an indicator of excessive thirst during the year before diagnosis caused by hyperglycemia) and risk of neither LADA (OR: 0.98, 95\% CI: 0.94-1.02 per daily serving) nor type 2 diabetes (OR: 0.99, 95\% CI: 0.96-1.03).

\section{Discussion}

High intake of sweetened beverages was associated with an increased risk of LADA; a two-fold increased risk was seen in consumers of more than two $200 \mathrm{~mL}$ servings per day, and each daily serving increment in sweetened beverage intake conferred a 15\% increased risk. High consumers also experienced a two-fold increased risk of type 2 diabetes and a $20 \%$ risk increase per serving, which is consistent with findings in a recent meta-analysis of prospective data (7). The associations were similar in separate analyses of sugar-sweetened and artificially sweetened beverages for the risk of LADA as well as type 2 diabetes, which has also been previously suggested for type 2 diabetes (8).

Proposed mechanisms by which sugar-sweetened beverage intake may increase diabetes risk are predominantly related to induced insulin resistance (29) and include decrease in hepatic insulin sensitivity (30), adverse effects of increased insulin demand due to high intermittent spikes in blood glucose levels (16) and increase in inflammatory markers such as C-reactive protein (31). Another possibility is that the increased risk is mediated by increases in overweight (32). In line with previous studies on type 2 diabetes $(1,3,5,7,8)$, the observed positive association remained after adjustment for BMI, possibly suggesting a direct adverse effect of sweetened beverage intake on glucose homeostasis. However, it could be that the BMI does not fully indicate the level of visceral fat, which is a predictor of type 2 diabetes (33). An effect on the development of autoimmunity is also possible. Sugar-sweetened beverages have previously been linked to type 1 diabetes (13) as well as rheumatoid arthritis (34), with suggestions of sugar being involved in causing infections and/or inflammation (34). In this study, high consumption of sweetened beverages was associated with insulin resistance and low beta cell function in type 2 diabetes patients, with similar tendencies in LADA, whereas no association with GADA levels was observed. A link between sugar-sweetened beverage intake and HOMA-IR was previously reported in adolescents $(35,36)$. Hence, the most plausible explanation may be that the excess risk of LADA among high consumers of sweetened beverages is related to mechanisms involving insulin resistance or other pathophysiological features shared with type 2 diabetes. The findings are also supportive of the accelerator hypothesis, in which it is postulated that beta cell stress caused by insulin resistance activates the immune system and consequently accelerates beta cell apoptosis leading to manifest diabetes. The intensity of the immune response, and hence the tempo of beta cell destruction, is suggested to be modulated by genetic susceptibility conferred by HLA genes (37).

We also found an excess risk in high consumers of artificially sweetened beverages, which is in line with previous studies in type 2 diabetes $(1,7)$. Although not containing sugars and thus not contributing to caloric intake, it has been suggested that artificially sweetened beverages may stimulate appetite, which may lead to positive energy balance and weight gain (38). Further, artificial sweeteners have also been hypothesized to have adverse effects on abdominal fat (39) and gut microbiota (12), which may induce glucose intolerance. Alternatively, one could speculate that consumers of artificially sweetened beverages may have swapped from sugarsweetened beverages to prevent further weight gain (39). In that case, we may actually be assessing diabetes risk related to previous high consumption of sugar-sweetened beverages. In line with this explanation, we found the highest BMI, both at present and age 20, in the group reporting high intakes of artificially sweetened beverages.

The main strength of this study is the populationbased design, with a large number of incident LADA patients, and the use of a FFQ that has been thoroughly validated against both biomarkers (28) and 24-h recall interviews (26), together with information on a wide variety of potential confounding factors. For sweetened beverage intake, correlation with diet records showed good agreement (A Wolk, unpublished results), but unfortunately there is no biomarker of such intake. Validation of the FFQ questions has been performed in another setting, and a number of years ago, however, the validation studies included both men and women who were all within the same age range as participants of this study.

The main limitation is the retrospective design that may lead to recall bias. However, for a rare disease like LADA, the case-control design is far more efficient than the corresponding prospective study, which would have required an unrealistically large sample and/or follow-up time to yield the same number of cases. In this study, patients were meticulously instructed to report their dietary habits as they were before diabetes diagnosis, but some patients may have reduced their 
consumption of sweetened beverages since diagnosis and report accordingly. Still, this would not lead us to overestimate the risk in high consumers, but rather the opposite. The fact that the patients were asked to recall their consumption of sweetened beverages as they were before diagnosis, whereas the controls were asked to recall their intake during the year directly before participation date, may introduce bias. In an attempt to minimize such recall bias, analysis were run separately for patients filling out the questionnaire within six months and three months of diagnosis, and excluded those who were treated by diet modification, but the results were similar. Some controls may have undiagnosed diabetes. This would make controls more similar to the cases with regard to lifestyle and hence dilute the true associations between intake of sweetened beverages and diabetes risk. Bias would be introduced if the participating controls are not representative of the population generating the cases, i.e. inhabitants of the counties of Scania and Uppsala, with regard to both sweetened beverage intake and other covariates. In a comparison using data of the general population, we found that the participating controls are equivalent with regard to educational level (data from Statistics Sweden, www.scb.se).

One could also speculate that the high consumption of sweetened beverages reported by patients reflects greater thirst in the period preceding diagnosis due to hyperglycemia. This is difficult to assess, but we noted that these patients did not report excess intake of water. Finally and most importantly for the credibility of these findings with regard to type 2 diabetes, our results were in line with previous reports based on prospective data with diet reported a several years before diagnosis $(1,3)$. False-positive LADA patients, i.e. truly type 2 diabetes (i.e. GADA negative) patients, but misclassified as LADA, could drive the results for LADA in the same direction as that of type 2 diabetes. However, this is unlikely to fully explain the observed association for LADA considering the high specificity of the GADA assay and the fact that the same magnitude of association was also observed among LADA patients with high GADA levels. Another explanation for our findings could be that high intake of sweetened beverages simply is a marker of an overall poor lifestyle leading onto the trajectory toward diabetes onset. We did observe that high consumers had an unhealthier lifestyle in general, and the highest BMI, both currently and at age 20 , and the greatest weight gain since age 20 . The excess risk in high consumers did persist after adjustment for a wide range of dietary and lifestyle factors including BMI, but we cannot rule out residual confounding due to unmeasured or imprecisely measured factors, such as BMI, energy intake or socio-demographic factors, as an explanation for our results. The group of high consumers constitutes $6 \%$ of the study participants, but it is noteworthy that among participants below age $50,12 \%$ consume such high levels.

In conclusion, these findings add support to the accumulating evidence suggesting that high intake of sweetened beverages, both sugar-sweetened and artificially sweetened, is a potential risk factor for type 2 diabetes. Importantly, these findings indicate that the adverse health effects seen with high sweetened beverage intake also encompass autoimmune forms of diabetes. The excess risk seems not to be fully explained by caloric intake or BMI, opening up for other explanations possibly including direct adverse effects of sweetened beverages on glucose homeostasis and insulin sensitivity. The suggested positive association between artificially sweetened beverages and diabetes risk remains to be further explored. This study supports the notion that dietary factors may influence LADA development $(21,40$, 41). This is important as the identification of modifiable risk factors could aid in preventing autoimmune diabetes. It is especially urgent in times when diabetes prevalence is on the rise (42) and sweetened beverage consumption continues to be high (43).

\section{Supplementary data}

This is linked to the online version of the paper at http://dx.doi.org/10.1530/ EJE-16-0376.

\section{Declaration of interest}

The authors declare that there is no conflict of interest that could be perceived as prejudicing the impartiality of the research reported.

\section{Funding}

The ESTRID study is financed by the Swedish Research Council (VR), the Swedish Research Council for Health, Working Life and Welfare (FORTE), AFA Insurance and the Swedish Diabetes Association. The Swedish Research Council is also supporting ANDIS (including ALF funding for clinical research in medicine) and ANDiU (including EXODIAB - strategic governmental funding for excellence of diabetes research in Sweden).

\section{Author contribution statement}

$J E L$ contributed to the design of the study, analyzed and interpreted the data and wrote the manuscript. S C researched data, contributed to the design of the study, interpreted the data and reviewed and revised the manuscript. $T$ A contributed to data analysis, interpretation of data and reviewing and revising of the manuscript. P-O C, M D, L $G$ and $M M$ researched data and contributed to reviewing and revising of the manuscript. T T and A W contributed to interpretation of data 
and reviewing and revising of the manuscript. All authors have read and approved the final version of the manuscript. J E L is the guarantor of the study taking responsibility for the integrity of the data and affirming that this is a truthful report of the study.

\section{References}

1 The InterAct Consortium. Consumption of sweet beverages and type 2 diabetes incidence in European adults: results from EPIC-InterAct. Diabetologia 201356 1520-1530. (doi: 10.1007/s00125-013-2899-8)

2 Palmer JR, Boggs DA, Krishnan S, Hu FB, Singer M \& Rosenberg L. Sugar-sweetened beverages and incidence of type 2 diabetes mellitus in African American women. Archives of Internal Medicine 2008168 1487-1492. (doi:10.1001/archinte.168.14.1487)

3 de Koning L, Malik VS, Rimm EB, Willett WC \& Hu FB. Sugar-sweetened and artificially sweetened beverage consumption and risk of type 2 diabetes in men. American Journal of Clinical Nutrition 201193 1321-1327. (doi:10.3945/ajcn.110.007922)

4 Schulze MB, Manson JE, Ludwig DS, Colditz GA, Stampfer MJ, Willett WC \& Hu FB. Sugar-sweetened beverages, weight gain, and incidence of type 2 diabetes in young and middle-aged women. JAMA 2004292 927-934. (doi:10.1001/jama.292.8.927)

5 Odegaard AO, Koh WP, Arakawa K, Yu MC \& Pereira MA. Soft drink and juice consumption and risk of physician-diagnosed incident type 2 diabetes: the Singapore Chinese Health Study. American Journal of Epidemiology 2010171 701-708. (doi:10.1093/aje/kwp452)

6 Ardisson Korat AV, Willett WC \& Hu FB. Diet, lifestyle, and genetic risk factors for type 2 diabetes: a review from the Nurses' Health Study, Nurses' Health Study 2, and Health Professionals' Follow-up Study. Current Nutrition Reports 20143 345-354. (doi: 10.1007/s13668014-0103-5)

7 Imamura F, O'Connor L, Ye Z, Mursu J, Hayashino Y, Bhupathiraju SN $\&$ Forouhi NG. Consumption of sugar sweetened beverages, artificially sweetened beverages, and fruit juice and incidence of type 2 diabetes: systematic review, meta-analysis, and estimation of population attributable fraction. BMJ 2015351 h3576. (doi:10.1136/bmj.h3576)

8 Greenwood DC, Threapleton DE, Evans CE, Cleghorn CL, Nykjaer C, Woodhead C \& Burley VJ. Association between sugar-sweetened and artificially sweetened soft drinks and type 2 diabetes: systematic review and dose-response meta-analysis of prospective studies. British Journal of Nutrition 2014112 725-734. (doi:10.1017/S0007114514001329)

9 Malik VS, Popkin BM, Bray GA, Després JP \& Hu FB. Sugarsweetened beverages, obesity, type 2 diabetes, and cardiovascular disease risk. Circulation 2010121 1356-1364. (doi:10.1161/ CIRCULATIONAHA.109.876185)

10 Nettleton JA, Lutsey PL, Wang Y, Lima JA, Michos ED \& Jacobs DR Jr. Diet soda intake and risk of incident metabolic syndrome and type 2 diabetes in the Multi-Ethnic Study of Atherosclerosis (MESA). Diabetes Care 200932 688-694. (doi:10.2337/dc08-1799)

11 Mattes RD \& Popkin BM. Nonnutritive sweetener consumption in humans: effects on appetite and food intake and their putative mechanisms. American Journal of Clinical Nutrition 200989 1-14. (doi:10.3945/ajcn.2008.26792)

12 Suez J, Korem T, Zeevi D, Zilberman-Schapira G, Thaiss CA, Maza O, Israeli D, Zmora N, Gilad S \& Weinberger A et al. Artificial sweeteners induce glucose intolerance by altering the gut microbiota. Nature 2014514 181-186. (doi:10.1038/nature13793)

13 Lamb MM, Frederiksen B. Seifert JA, Kroehl M, Rewers M \& Norris JM Sugar intake is associated with progression from islet autoimmunity to type 1 diabetes: the Diabetes Autoimmunity Study in the Young. Diabetologia 201558 2027-2034. (doi:10.1007/s00125-015-3657-x)

14 Pundziute-Lyckå A, Persson LA, Cedermark G, Jansson-Roth A, Nilsson U, Westin V \& Dahlquist G. Diet, growth, and the risk for type 1 diabetes in childhood: a matched case-referent study. Diabetes Care 200427 2784-2789. (doi: 10.2337/diacare.27.12.2784)
15 Eizirik DL \& Darville MI. $\beta$-Cell apoptosis and defense mechanisms: lessons from type 1 diabetes. Diabetes 200150 S64-S69. (doi:10.2337/ diabetes.50.2007.S64)

16 Shao C, Gu J, Meng X, Zheng H \& Wang D. Systematic investigation into the role of intermittent high glucose in pancreatic beta-cells. International Journal of Clinical and Experimental Medicine 20158 5462-5469.

17 Kohnert KD, Freyse EJ \& Salzsieder E. Glycaemic variability and pancreatic $\beta$-cell dysfunction. Current Diabetes Reviews 20128 345-354. (doi:10.2174/157339912802083513)

18 Björk E, Kämpe O, Karlsson FA, Pipeleers DG, Andersson A, Hellerström C \& Eizirik DL. Glucose regulation of the autoantigen GAD65 in human pancreatic islets. Journal of Clinical Endocrinology and Metabolism 199275 1574-1576. (doi: 10.1210/jc.75.6.1574)

19 Tuomi T, Santoro N, Caprio S, Cai M, Weng J \& Groop L. The many faces of diabetes: a disease with increasing heterogeneity. Lancet 2014 383 1084-1094. (doi:10.1016/S0140-6736(13)62219-9)

20 Hawa MI, Kolb H, Schloot N, Beyan H, Paschou SA, Buzzetti R, Mauricio D, De Leiva A, Yderstraede K \& Beck-Nielsen H et al. Adultonset autoimmune diabetes in Europe is prevalent with a broad clinical phenotype: action LADA 7. Diabetes Care 201336 908-913. (doi:10.2337/dc12-0931)

21 Löfvenborg JE, Andersson T, Carlsson PO, Dorkhan M, Groop L, Martinell M, Rasouli B, Storm P, Tuomi T \& Carlsson S. Coffee consumption and the risk of latent autoimmune diabetes in adults - results from a Swedish case-control study. Diabetic Medicine 201431 799-805. (doi: 10.1111/dme.12469)

22 Lund University. ANDIS - Alla Nya Diabetiker I Skåne. 2016. (available at: http://andis.ludc.med.lu.se/). Accessed on August 2016

23 Vandenbroucke JP \& Pearce N. Case-control studies: basic concepts. International Journal of Epidemiology 201241 1480-1489. (doi:10.1093/ ije/dys147)

24 Rahmati K, Lernmark A, Becker C, Foltyn-Zadura A, Larsson K, Ivarsson SA \& Törn C. A comparison of serum and EDTA plasma in the measurement of glutamic acid decarboxylase autoantibodies (GADA) and autoantibodies to islet antigen-2 (IA-2A) using the RSR radioimmunoassay (RIA) and enzyme linked immunosorbent assay (ELISA) kits. Clinical Laboratory 200854 227-235.

25 The Oxford Center for Diabetes. Endocrinology \& Metabolism. Diabetes Trial Unit. HOMA Calculator. (available from: http://www. dtu.ox.ac.uk/homacalculator/index.php). Accessed on June 2013.

26 Messerer M, Johansson SE \& Wolk A. The validity of questionnairebased micronutrient intake estimates is increased by including dietary supplement use in Swedish men. Journal of Nutrition 2004134 1800-1805.

27 Levitan EB, Westgren CW, Liu S \& Wolk A. Reproducibility and validity of dietary glycemic index, dietary glycemic load, and total carbohydrate intake in 141 Swedish men. American Journal of Clinical Nutrition 200785 548-553.

28 Wallin A, Di Giuseppe D, Burgaz A, Håkansson N, Cederholm T, Michaëlsson K \& Wolk A. Validity of food frequency questionnairebased estimates of long-term long-chain n-3 polyunsaturated fatty acid intake. European Journal of Nutrition 2014 53 549-555. (doi:10.1007/s00394-013-0563-1)

29 Otero-Losada M, Cao G, González J, Muller A, Ottaviano G, Lillig C, Capani F, Ambrosio G \& Milei J. Functional and morphological changes in endocrine pancreas following cola drink consumption in rats. PLOS ONE 201510 e0118700. (doi:10.1371/journal.pone.0118700)

30 Maki KC, Nieman KM, Schild AL, Kaden VN, Lawless AL, Kelley KM \& Rains TM. Sugar-sweetened product consumption alters glucose homeostasis compared with dairy product consumption in men and women at risk of type 2 diabetes mellitus. Journal of Nutrition 2015 145 459-466. (doi:10.3945/jn.114.204503)

31 Liu S, Manson JE, Buring JE, Stampfer MJ, Willett WC \& Ridker PM. Relation between a diet with a high glycemic load and plasma concentrations of high-sensitivity C-reactive protein in middle-aged women. American Journal of Clinical Nutrition 200275 492-498. 
32 Malik VS, Pan A, Willett WC \& Hu FB. Sugar-sweetened beverages and weight gain in children and adults: a systematic review and meta-analysis. American Journal of Clinical Nutrition 201398 1084-1102. (doi:10.3945/ajcn.113.058362)

33 Hanley AJ, Wagenknecht LE, Norris JM, Bryer-Ash M, Chen YI, Anderson AM, Bergman R \& Haffner SM. Insulin resistance, beta cell dysfunction and visceral adiposity as predictors of incident diabetes: the Insulin Resistance Atherosclerosis Study (IRAS) Family study. Diabetologia 200952 2079-2086. (doi:10.1007/s00125-009-1464-y)

$34 \mathrm{Hu}$ Y, Costenbader KH, Gao X, Al-Daabil M, Sparks JA, Soloman DH, $\mathrm{Hu}$ FB, Karlson EW \& Lu B. Sugar-sweetened soda consumption and risk of developing rheumatoid arthritis in women. American Journal of Clinical Nutrition 2014100 959-967. (doi:10.3945/ ajcn.114.086918)

35 Bremer AA, Auinger P \& Byrd RS. Relationship between insulin resistanceassociated metabolic parameters and anthropometric measurements with sugar-sweetened beverage intake and physical activity levels in US adolescents: findings from the 1999-2004 National Health and Nutrition Examination Survey. Archives of Pediatrics and Adolescent Medicine 2009 163 328-335. (doi:10.1001/archpediatrics.2009.21)

36 Kondaki K, Grammatikaki E, Jiménez-Pavón D, De Henauw S, González-Gross M, Sjöstrom M, Gottrand F, Molnar D, Moreno LA \& Kafatos A et al. Daily sugar-sweetened beverage consumption and insulin resistance in European adolescents: the HELENA (Healthy Lifestyle in Europe by Nutrition in Adolescence) Study. Public Health Nutrition 201316 479-486. (doi:10.1017/S1368980012002613)
37 Wilkin TJ. The accelerator hypothesis. Pediatric Diabetes 201213 334-339. (doi:10.1111/j.1399-5448.2011.00831.x)

38 Blundell JE \& Hill AJ. Paradoxical effects of an intense sweetener (aspartame) on appetite. Lancet 19861 1092-1093. (doi:10.1016/ S0140-6736(86)91352-8)

39 Pereira MA. Diet beverages and the risk of obesity, diabetes, and cardiovascular disease: a review of the evidence. Nutrition Reviews 201371 433-430. (doi:10.1111/nure.12038)

40 Rasouli B, Andersson T, Carlsson PO, Dorkhan M, Grill V, Groop L, Martinell M, Tuomi T \& Carlsson S. Alcohol and the risk for latent autoimmune diabetes in adults: results based on Swedish ESTRID study. European Journal of Endocrinology 2014171 535-543. (doi:10.1530/EJE-14-0403)

41 Löfvenborg JE, Andersson T, Carlsson PO, Dorkhan M, Groop L, Martinell M, Tuomi T, Wolk A \& Carlsson S. Fatty fish consumption and risk of latent autoimmune diabetes in adults. Nutrition and Diabetes 20144 e139. (doi: 10.1038/nutd.2014.36)

42 World Health Organization. Global Report on Diabetes. 2016. (available at: http://apps.who.int/iris/bitstream/10665/204871/1/9789241565257_ eng.pdf?ua=1). Accessed on April 2016.

43 Singh GM, Micha R, Khatibzadeh S, Shi P, Lim S, Andrews KG, Engell RE, Ezzati M, Mozaffarian D \& Global Burden of Diseases Nutrition and Chronic Diseases Expert Group (NutriCoDe). Global, regional, and national consumption of sugar-sweetened beverages, fruit juices, and milk: a systematic assessment of beverage intake in 187 countries. PLoS ONE 201510 e0124845. (doi:10.1371/journal.pone.0124845)

Received 29 April 2016

Revised version received 17 August 2016

Accepted 15 September 2016 